\title{
C-REACTIVE PROTEIN - A RELIABLE PREDICTIVE PARAMETER IN EXPULSION OF SMALL DISTAL URETERIC CALCULUS
}

\author{
Manjunath Shettyㄹ, Siva Sai Bharadwaja², Antony Thomas ${ }^{3}$, Mohan Suvarna ${ }^{4}$ \\ 1 Professor, Department of Urology, Father Muller Medical College, Mangalore. \\ ${ }^{2}$ Resident, Department of Urology, Father Muller Medical College, Mangalore. \\ ${ }^{3}$ Resident, Department of Urology, Father Muller Medical College, Mangalore. \\ 4 Professor, Department of Urology, Father Muller Medical College, Mangalore.
}

\section{ABSTRACT}

\section{BACKGROUND}

Plasma C-Reactive Protein (CRP) is an acute-phase protein whose serum level increases in response to inflammation, as in impacted ureteric calculus.

\section{MATERIALS AND METHODS}

Patients with distal ureteric calculus between $5-7 \mathrm{~mm}$ size were included in this study. All patients were subjected to history taking, x-ray KUB, urinary tract ultrasound, non-contrast CT (NC-CTKUB) scan and plasma CRP estimation. All patients received medical expulsive therapy. Patients were monitored on a weekly basis using ultrasound until spontaneous calculus passage or intervention, for 4 weeks. Patients who failed to expel the calculus within 4 weeks underwent ureteroscopy and removal.

\section{RESULTS}

Spontaneous expulsion of within 4 weeks was recorded in 294 patients (Group 1), while 241 patients (Group 2) underwent ureteroscopy for stone extraction. Patients with spontaneous stone expulsion had significantly lower serum CRP levels $(16.45+$ 2.58) than those who failed to pass the stone spontaneously $(39.67+6.30)$. Receiver operator characteristic curve is used to determine CRP cut-off point for prediction of spontaneous ureteric stone expulsion. A cut-off point of $21.9 \mathrm{mg} / \mathrm{L}$ for CRP yielded appeared optimal for prediction of spontaneous ureteric stone expulsion.

\section{CONCLUSION}

Medical expulsive therapy success for management of small distal ureteric calculi could be predicted with plasma CRP. Patients with CRP > $21.9 \mathrm{mg} / \mathrm{L}$ have low stone expulsion rate and should directly be subjected for an immediate, minimally invasive ureteroscopy.

\section{KEYWORDS}

CRP, Distal Ureteric Calculus.

HOW TO CITE THIS ARTICLE: Shetty M, Bharadwaja SS, Thomas A, et al. C-reactive protein - a reliable predictive parameter in expulsion of small distal ureteric calculus. J. Evolution Med. Dent. Sci. 2016;5(96):7091-7093, DOI: 10.14260/jemds/2016/1604

\section{BACKGROUND}

The incidence of urolithiasis is estimated to be about 5 - 15\% worldwide. $(1,2)$ Ureteric calculus represent about $20 \%$ of the incidence. Stone size is the most important parameter to predict the possibility of the spontaneous passage and is one point to impress the patient upon the spontaneous passage or need for intervention. The incidence of spontaneous passage of distal ureteric calculus $1 \mathrm{~mm}$ diameter was $87 \%, 2-4 \mathrm{~mm}$ $76 \%, 5-7 \mathrm{~mm} 60 \%$ and $7-9 \mathrm{~mm} \mathrm{48 \%}$ and more than $9 \mathrm{~mm}$ is less than $25 \% \cdot{ }^{(1,2)}$ Sometimes simple conservative management could lead on to complications, hence a need for a parameter to convince the patient regarding the need for intervention in order to avoid complications like urinary tract infections, obstruction and hydronephrosis and deterioration in renal function and urosepsis. $(3,4,5)$ Serum C-reactive protein is a non-specific marker of systemic inflammation. Here, we investigated the relationship between serum CRP and expulsion of the calculus.

Financial or Other, Competing Interest: None.

Submission 26-10-2016, Peer Review 18-11-2016,

Acceptance 25-11-2016, Published 01-12-2016.

Corresponding Author:

Dr. Manjunath Shetty,

Professor, Department of Urology

Father Muller Medical College, Mangalore.

E-mail:manjunathshetty51@gmail.com

DOI: $10.14260 /$ jemds/2016/1604
C-Reactive Protein (CRP) is a protein found in the blood, the levels of which rise in response to inflammation (i.e. it is an acute-phase protein). Its physiological role is to bind to phosphocholine expressed on the surface of dead or dying cells (and some types of bacteria) in order to activate the complement system.[6] CRP is synthesised by the liver.[6] Because there are a large number of conditions that can increase CRP production, an elevated CRP level does not diagnose a specific disease. CRP has been proven to be useful in several clinical urological conditions such as estimation of renal injury in pyelonephritis,[7] evaluation of the severity of urinary tract infection in children[7] and even avoiding voiding cystourethrography in children with febrile urinary tract infection and vesicoureteral reflux.[7]

The aim of our study is to assess the usefulness of CRP as a new biochemical to assist in making a decision concerning intervention versus observation of ureteric stones.

\section{MATERIALS AND METHODS}

The population of our study was composed of patients with distal ureteric calculi, size ranging from $5-7 \mathrm{~mm}$.

\section{Inclusion Criteria}

All male and female patients older than 18 years were eligible for inclusion in this study. 


\section{Exclusion Criteria}

Patients with urinary tract infection, severe hydronephrosis, multiple ureteral stones, pregnancy, solitary kidney, impaired renal function, associated ureteric anomaly, previous ureteric surgery or endoscopic procedures, painful symptoms experienced for more than 1 day and the patient's wish to remove the stone immediately. Patients suffering from any inflammatory disease (Viral infection, arthritis, gastroenteritis, hepatitis or respiratory infection), active neoplasia, cardiovascular disease (including hypertension), overweight/obesity, diabetes or liver failure are excluded from this study. Both inflammatory and neoplastic conditions may elevate CRP and conversely hepatic disease may lead to impaired CRP synthesis. Additionally, we exclude patients with history of certain medications as statins, steroids and oral contraceptives that affect CRP.

All eligible patients were informed about the opportunity to be recruited into the trial. Ureteroscopy was suggested as an alternative therapeutic option.

All patients were subjected to history taking, KUB, ultrasound and Non-Contrast CT (NC-CTKUB) scan to diagnose stone site and size. Non-Contrast CT (NC-CTKUB) scan is internationally accepted as gold standard for the investigation and measurement of ureteric calculi.

CRP values checked only upon initial presentation along with the renal parameters (before use of diclofenac and other NSAIDs, as these drugs reduce CRP). Serum CRP was measured by dilution method using a latex agglutination test, AVITEX CRP (Omega Diagnostics Ltd., Alloa, Scotland, UK). The method used was that described by the manufacturers. It was performed by mixing equal volumes of latex reagents with undiluted sera of patients. Normal CRP level in our laboratory is $<6 \mathrm{mg} / \mathrm{L}$. Level above $6 \mathrm{mg} / \mathrm{L}$ were considered as high.

All patients received alfuzosin once daily and they were allowed to use symptomatic therapy with injections of 50 mgs of Tramadol (on demand). In addition, all subjects were instructed to drink $2.5 \mathrm{~L}$ of water daily. To observe possible stone expulsion, all patients were required to filter the urine. All patients were examined weekly using KUB and urinary tract ultrasound until spontaneous stone passage or intervention after 4 weeks. Patients who failed to expel the stone within 4 weeks underwent ureteroscopy.

\section{RESULTS}

A total of 535 patients were included in the study. The mean age of patients was 35.8 years and the male-to-female ratio was 1.2:1. Spontaneous stone expulsion within 4 weeks was recorded in 294 patients (Group 1) (54.9\%) and 241 patients (Group 2) (45.1\%) underwent ureteroscopy for stone extraction because of failure of spontaneous stone expulsion, urosepsis in 2 patients and uncontrollable pain in 1 patient.

In (Group 1) 198 (67.4\%) patients out of 294 stone passers captured the stone as documentation of passage; spontaneous stone expulsion is diagnosed radiologically in 96 (32\%) patients out of 294 stone passers.

In (Group 2) during ureteroscopy, inflammatory ureteral polyps is observed at the stone site in 25 patients $(10.4 \%)$ and a ureteral stricture adjacent to the stone in 18 patients (7.6\%). No lesions were observed in 198 patients (82\%).

Patients with spontaneous stone expulsion had significantly lower serum CRP levels $(16.45+2.58)$ than those who failed to pass the stones spontaneously $(39.67+$ 6.30). During the period of medical expulsive therapy for distal ureteric stone, 6 patients in Group 1 (2.1\%) and 11 patients in Group 2 (4.5\%) needed an emergency room visit because of painful symptoms that could not be controlled at home using the analgesic prescribed $(\mathrm{p}=0.089)$. The treatment-related side effects were generally mild and included dizziness, rhinitis and abnormal ejaculation, which reported in 18 patients in Group 1 (6.1\%) and 15 patients in Group $2(6.2 \%)(p=0.365)$.

An analysis of patient's data revealed that the patient's age, sex and laterality did not differ between both groups. Stone size was comparable in both groups. Using a regression analysis, CRP $(<0.003)$ was found to be an independent predictive factor for spontaneous ureteric stone expulsion.

Receiver operator characteristic curve is used to determine CRP cut-off point for prediction of spontaneous ureteric stone expulsion. A cut-off point of $21.9 \mathrm{mg} / \mathrm{L}$ for CRP yielded, appeared optimal for prediction of spontaneous ureteric stone expulsion (Sensitivity $78.6 \%$, specificity $89.3 \%$, positive predictive value $85.1 \%$ and negative predictive value $77.4 \%)$.

\section{DISCUSSION}

Ureteral stones affect $15 \%$ of the population and are commonly diagnosed at emergency rooms[1] and urologists are frequently asked to choose an effective and safe therapy. Ureteroscopy represent the current mini-invasive therapeutic options for ureterolithiasis. [5] Nevertheless, these procedures are not risk-free, are problematic and are quite expensive. Observation of ureteral calculi, although attractive since it avoids invasive procedures is associated with pain, uncertainty, potential risks to renal function, urinary tract infection and occasionally loss of work. Therefore, it is difficult to choose between minimally invasive therapies and a watchful-waiting approach, especially when patients report few symptoms and/or stones are small.(5,6)

Medical therapy with alpha blockers like Alfuzosin or Tamsulosin proved to induce stone expulsion and to relieve ureteral colic pain as shown by significantly less analgesic use, consequently determining the possibility of good home patient management and a loss of fewer working days. Recently others have observed that a conservative approach to ureteral stones is associated with lower cost compared with any invasive procedures (Ureteroscopy or extracorporeal shock wave lithotripsy) only if it is successful that is followed by stone expulsion. ${ }^{(8,9)}$ In fact, failure of conservative therapy results in higher costs than for first line ureteroscopy since it implies the loss of more working days as well as the need of a larger number of urgent urological visits. Patients who were not stone-free after the 4-week followup were successfully treated with ureteroscopy. These data demonstrate that neither watchful waiting nor medical therapy seems to negatively affect the success rate of stone removal. $(5,6)$

The decision to proceed with intervention (Ureteroscopy) or to continue observation of small ureteral calculi is complex. Data suggests that stone size influences the spontaneous stone expulsion rate. ${ }^{[5,8,9]}$ Patient factors that must be considered include degree of pain, narcotic requirements, work requirements, family commitments and personal preference. 
In this study, we estimated serum CRP level in patients with ureteric stone as a new parameter to assist in making a decision concerning intervention versus observation. It is not surprising then that CRP level may be a diagnostic tool for some urological diseases, such as for differentiating acute appendicitis from right-sided renal colic[10] and acute pyelonephritis from cystitis[11] for predicting the development of infection in patients who have undergone lithotripsy ${ }^{[2,13]}$ or for diagnosing and following up urinary tract infections in children.[14]

Serum CRP level is a nonspecific marker of systemic inflammation. It is protein synthesised mainly in the liver and its determination of serum CRP may reflect the intensity of the inflammatory or infectious process. $(8,14)$

It has been shown that larger calculi particularly tend to provoke intense inflammatory changes in the ureteric wall and that submucosal oedema in proximity to a stone may worsen ureteric obstruction, heightening the risk of impaction and retention. ${ }^{(10)}$

According to some studies, which investigate the role of CRP as a diagnostic marker in some urological diseases $(6,7)$ and depending on the ureteric wall inflammation induced by ureteric stones,(10) we evaluate serum CRP level in patients with ureteric stones in a trial to use CRP as a factor that assist in management of ureteric stones in addition to other factors as stone size, site and degree of hydronephrosis. In our study, we reported that a significant association between spontaneous ureteric stone expulsion and serum CRP level in our study implies that inflammatory changes in the ureteric wall and that submucosal oedema in association with ureteric stone may play a role in failure of medical expulsive therapy of ureteric stone.(10) We noticed that serum CRP is significantly higher in patients who failed to expel the stone within 4 weeks than in those with spontaneous stone expulsion within 4 weeks $(39.67+6.30$ and $16.45+2.58$ respectively, $\mathrm{p}$ value $=0.001$ ). The patient's age, sex and laterality did not differ between both groups. Stone size was comparable in both groups.

Using ROC curve in our study, a cut-off point of $21.9 \mathrm{mg} / \mathrm{L}$ for CRP yielded, appeared optimal for prediction of spontaneous ureteric stone expulsion (sensitivity 78.6\%, specificity $89.3 \%$, positive predictive value $85.1 \%$ and negative predictive value $77.4 \%$ ).

We believe that CRP could be added as one of the factors that the urologist must consider when recommending treatment to patients with ureteral calculi (e.g. stone location, size, degree of hydronephrosis, UTI and symptom severity. $(1,2,5,14)$

We conclude that CRP status might predict the likelihood of stone passage without intervention, indeed larger prospective trials are required to validate this hypothesis and provide consideration with appropriate statistical analyses of the above-cited potential confounders that alter CRP value. This is especially relevant as many, if not most, patients with urinary tract stones have one or more of the conditions above.

\section{CONCLUSION}

Medical expulsive therapy success for management of small distal ureteric calculi could be predicted with serum CRP value. Patients with CRP > $21.9 \mathrm{mg} / \mathrm{L}$ have low stone expulsion rate and should directly be subjected for an immediate, minimally invasive ureteroscopy and extraction of the calculus.

\section{REFERENCES}

1. Lotan Y, Gettman MT, Roehrborn CG, et al. Management of ureteral calculi: a cost comparison and decision making analysis. J Urol 2002;167(4):1621-9.

2. Ueno A, Kawamura T, Ogawa A, et al. Relation of spontaneous passage of ureteral calculi to size. Urology 1977;10(6):544-6.

3. Coll DM, Varanelli MJ, Smith RC. Relationship of spontaneous passage of ureteral calculi to stone size and location as revealed by unenhanced helical CT. AJR Am J Roentgenol 2002;178(1):101-3.

4. Pepys MB, Hirschfield GM. C-reactive protein: a critical update. J Clin Invest 2003;111(12):1805-12.

5. Segura JW, Preminger GM, Assimos DG, et al. Ureteral stones clinical guidelines panel summary report on the management of ureteral calculi. The American Urological Association. J Urol 1997;158(5):1915-21.

6. Pulliam PN, Attia MW, Cronan KM. C-reactive protein in febrile children aged 1 to 36 months of age with clinically undetectable serious bacterial infection. Pediatrics 2001;108(6):1275-79.

7. Horcajada JP, Velasco M, Filella X, et al. Evaluation of inflammatory and renal-injury markers in women treated with antibiotics for acute pyelonephritis caused by Escherichia coli. Clin Diagn Lab Immunol 2004;11(1):142-6.

8. Miller OF, Kane CJ. Time to stone passage for observed ureteral calculi: a guide for patient education. J Urol 1999;162(3 Pt 1):688-91.

9. Ibrahim AI, Shetty SD, Awad RM, et al. Prognostic factors in the conservative treatment of ureteric stones. Br J Urol 1991;67(4):358-61.

10. Yamaguchi K, Minei S, Yamazaki T, et al. Characterization of ureteral lesions associated with impacted stones. Int J Urol 1999;6(6):281-5.

11. Paajanen $H$, Tainio H, Laato $M$. A chance of misdiagnosis between acute appendicitis and renal colic. Scand J Urol Nephrol 1996;30(5):363-6.

12. Dellabella M, Milanese G, Muzzonigro G. Efficacy of tamsulosin in the medical management of juxtavesical ureteral stones. J Urol 2003;170(6 Pt 1):2202-5.

13. Yilmaz E, Batislam E, Tuglu D, et al. C-reactive protein in early detection of bacteremia and bacteriuria after extracorporeal shock wave lithotripsy. Eur Urol 2003;43(3):270-4.

14. Shaoul R, Lahad A, Tamir A, et al. C-reactive protein (CRP) as a predictor for true bacteremia in children. Med Sci Monit 2008;14(5):CR255-CR261. 